VoL. $50(1994) \quad[109-116]$

\title{
ON THE PETTIS MEASURABILITY THEOREM
}

\section{Dietrich Helmer}

It is shown that, in Pettis's criterion for Bochner measurability of a vector-valued function $f: S \rightarrow X$, scalar measurability of $f$ can be weakened to requiring that $u$ of be measurable for $u$ in some subset of the dual $X^{*}$ separating the points of $X$. Even then, the separability hypotheses in Pettis's Theorem can be weakened as well.

Throughout, $(S, \Sigma, \mu)$ denotes a positive measure space. Then $\Sigma_{0}$ stands for the collection of all $\mu$-null sets and $\Sigma_{b}$ for $\{E \in \Sigma \mid 0<\mu(E)<\infty\}$. Moreover, $X$ is a Fréchet space over $\mathbb{K}$, where $\mathbb{K}=\mathbb{R}$ or $\mathbb{K}=\mathbb{C}$. We fix a function $f: S \rightarrow X$. Recall that $f$ is $\mu$-measurable (in the sense of Bochner) if and only if $\chi_{E} f$ is, for every $E \in \Sigma_{b}$, the limit $\mu$-almost everywhere in $S$ of a sequence of $\mu$-simple functions. The set of all $\mu$-measurable functions $S \rightarrow X$ is denoted by $\mathcal{M}(\mu, X)$.

One of the most frequently employed and useful criteria for $\mu$-measurability is the Pettis Measurability Theorem for Banach spaces $X[12$, p.278] (compare [2, p.149]): $f \in \mathcal{M}(\mu, X)$ if (and only if) every $E \in \Sigma_{b}$ admits an $N \in \Sigma_{0}$ such that $f(E \backslash N)$ is separable and $f$ is scalarly $\mu$-measureable, that is,

$$
X^{*} \circ f:=\left\{u \circ f \mid u \in X^{*}\right\} \subseteq \mathcal{M}(\mu, \mathbb{K})=: \mathcal{M}(\mu)
$$

A discussion of the importance of the theorem for Measure Theory and the theory of Banach spaces has been given by Uhl in [19]. It is known (compare [19] [1, p.43] for finite $\mu$ ) that scalar $\mu$-measurability of $f$ in Pettis's Theorem can be replaced by the weaker condition that $U \circ f \subseteq \mathcal{M}(\mu)$ for some norming $U \subseteq X^{*}$. We shall show in this note that a minimal requirement is already sufficient here: namely, that $U$ of $\subseteq \mathcal{M}(\mu)$ for some $U \subseteq X^{*}$ separating sufficiently many points of $X$. (A key argument has already been utilised implicitly in [4] for Haar measures $\mu$ and, for general $\sigma$-finite $\mu$, in [7].) It is often easy to identify point-separating subsets $U$ of $X^{*}$; and, depending on $X^{*}$ and $f$, there may be flexibility in tailoring $U$ so as to facilitate the verification of $U \circ f \subseteq \mathcal{M}(\mu)$.

Let II:=[0,1]. If $B \subseteq X$, then $\overline{\operatorname{span}} B$ stands for the closed linear span of $B$ in $X$ and $B_{\sigma}$ denotes $B$ with the subspace topology it inherits from the weak topology of $X$.

Received 5th October, 1993

Copyright Clearance Centre, Inc. Serial-fee code: 0004-9729/94 \$A2.00+0.00. 
Similarly, if $V \subseteq X^{*}$, then $V_{\sigma}$ is $V$ equipped with the restricted $w^{*}$-topology. $(S, \widehat{\Sigma}, \mu)$ denotes the Lebesgue extension of $(S, \Sigma, \mu)$. We call $\Sigma_{c} \subseteq \widehat{\Sigma} \mu$-covering if and only if for every $E \in \Sigma_{b}$, there exists some $T \in \Sigma_{c}$ such that $E \cap T \notin \Sigma_{0}$, which is the case if and only if every $E \in \Sigma_{b}$ admits a sequence $T_{n}$ in $\Sigma_{c}$ with $E \backslash \bigcup_{n=1}^{\infty} T_{n} \in \Sigma_{0}$. And $\Gamma \subseteq \widehat{\Sigma}$ is said to be a $\mu$-regularity set if and only if $\mu(E)=\sup \{\mu(G) \mid G \in \Gamma, G \subseteq E\}$ for every $E \in \Sigma_{b}$. Any such $\Gamma$ is $\mu$-covering.

THEOREM 1. $f \in \mathcal{M}(\mu, X)$ if and only if there exists a $\mu$-covering set $\Sigma_{c}$ such that every $T \in \Sigma_{c}$ admits some $N \in \Sigma_{0}$ with $f(T \backslash N)$ separable and some $U \subseteq X^{*}$ separating the points of span $f(T \backslash N)$ with $U \circ f \subseteq \mathcal{M}(\mu)$.

Proof: If $f$ is $\mu$-measurable, then the conditions are satisfied with $\Sigma_{c}:=\Sigma_{b}$ and $U:=X^{*}$. Conversely, suppose now that there exists a $\mu$-covering collection $\Sigma_{c}$ as above. First, using routine arguments, one reduces the proof to the case where $\mu$ is finite, $X$ is separable, and where $U \circ f \subseteq \mathcal{M}(\mu)$ for some subset $U$ of $X^{*}$ separating the points of $X$. In this situation, it follows by means of Egoroff's Theorem that the linear subspace $D:=\left\{u \in X^{*} \mid u \circ f \in \mathcal{M}(\mu)\right\}$ of $X^{*}$ is sequentially closed in $X_{\sigma}^{*}$. For every 0-neighbourhood $B$ in $X$, the polar $B^{\circ}:=\left\{u \in X^{*}|| u(b) \mid \leqslant 1\right.$ for all $\left.b \in B\right\}$ is compact and metrisable in $X_{\sigma}^{*}$; and, consequently, $D \cap B^{\circ}$ is closed in $\left(B^{\circ}\right)_{\sigma}$. According to the Krein-Šmulian Theorem $[9,22.6]$, therefore, $D$ itself is closed in $X_{\sigma}^{*}$. On the other hand, $D$ is dense in $X_{\sigma}^{*}$ since it separates the points of $X$. Thus, $D=X^{*}$, that is $f$ is scalarly $\mu$-measurable. If $X$ is a Banach space, an appeal to Pettis's Theorem finishes the proof. In the general case, the usual arguments need adaptation: Fix a sequence $B_{n}$ of closed convex circled subsets of $X$ constituting a 0-neighbourhood base in $X$ such that $B_{n+1} \subseteq B_{n}$ for all $n$. Thereafter, pick, for every $n$, a sequence $u_{n k}$ that is dense in $\left(B_{n}^{\circ}\right)_{\sigma}$. Moreover, let $x_{m}$ be a dense sequence in $X$. Then

$$
h_{n m}: s \longmapsto \sup \left\{\left|u_{n k}\left(f(s)-x_{m}\right)\right| \mid k \in \mathbb{N}\right\}: S \longrightarrow \mathbb{K}
$$

is $\mu$-measurable for all $n, m$, and, consequently, $h_{n m}^{-1}(\mathbb{I}) \in \widehat{\Sigma}$. But $h_{n m}^{-1}(\mathbb{I})=$ $f^{-1}\left(x_{m}+B_{n}\right)$ by means of the Bipolar Theorem. Now the classical arguments carry over mutatis mutandis to show that $f$ is the uniform limit of a sequence $f_{n}$ of countablyvalued functions with $\left\{f_{n}^{-1}(x) \mid n \in \mathbb{N}, x \in X\right\} \subseteq \widehat{\Sigma}$ and, finally, to show that $f$ is $\mu$-measurable.

EXAMple. Suppose $S$ is also a topological space and $\Gamma$ is a $\mu$-regularity set consisting of closed subsets or of open subsets of $S$. Let $D$ be an open connected set in $\mathbb{C}$ and $h: S \times D \rightarrow \mathbb{C}$ a function that is analytic in the second argument such that $\{h(-, z) \mid z \in A\} \subseteq \mathcal{M}(\mu)$ for some $A \subseteq D$ with an accumulation point in $D$. Then, given $\varepsilon>0$, every $E \in \Sigma_{b}$ contains a $G \in \Gamma$ with $\mu(E \backslash G)<\varepsilon$ such that $h$ is continuous on $G \times D$. 
Proof: Consider $\psi: s \mapsto h(s,-): S \rightarrow H(D)$, where $H(D)$ denotes the separable Fréchet space consisting of all analytic functions $D \rightarrow \mathbb{C}$ equipped with the topology of uniform convergence on compacta. The subset $U$ of $H(D)^{*}$, made up of the evaluation functionals at points of $A$, separates the points of $H(D)$ and satisfies $U \circ \psi \subseteq \mathcal{M}(\mu)$. So $\psi$ is $\mu$-measurable by Theorem 1; whence some sequence of countably-valued $\mu$-measurable functions $S \rightarrow H(D)$ converges to $\psi$ uniformly. Adapting standard arguments (compare [16, p.27]), given $\varepsilon$ and $E$, we find some $G \in \Gamma$ with $G \subseteq E$ and $\mu(E \backslash G)<\varepsilon$ on which $\psi$ is continuous. To complete the proof, it therefore suffices to utilise the continuity of the evaluation $H(D) \times D \rightarrow \mathbb{C}$.

We now turn to the second ingredient of the Pettis Theorem, separability, and replace it by weaker conditions, while keeping our minimal hypotheses on scalar $\mu$ measurability.

We call a topology $\mathcal{T}$ on $R \subseteq S$ a $\mu$-topology if and only if $\mathcal{T} \cap \Sigma_{0} \subseteq\{\emptyset, R\}$. If $R$ is $\sigma$-finite in $\widehat{\Sigma}$ and $\theta$ is a lifting of $\Delta:=\{D \in \widehat{\Sigma} \mid D \subseteq R\}$, or just any lower density of $\Delta$, then the density topology

$$
\mathcal{T}_{\theta}:=\left\{\theta(D) \backslash M \mid D \in \Delta, M \in \Delta \cap \Sigma_{0}\right\}=\{F \in \Delta \mid F \subseteq \theta(F)\}
$$

(compare [8, p.54]) is a $\mu$-topology on $R$. Generally: if $R \in \widehat{\Sigma} \backslash \Sigma_{0}$ and $\mathcal{O}$ is any topology on $R$ with $\mathcal{O} \cap \Sigma_{0}=\{\emptyset\}$, then $\left\{Q \backslash M \mid Q \in \mathcal{O}, M \in \Sigma_{0}\right\}$ is a basis of a $\mu$-topology. If $P$ is a topological space and $V \subseteq C(P)$, then $V$ is considered equipped with the topology of pointwise convergence and $A \lg V$ denotes the smallest closed $\mathbb{K}$ subalgebra of $C(P)$ containing $V$. Recall that $P$ satisfies the Souslin countable chain condition CCC if and only if every family of non-empty, pairwise disjoint, open subsets of $P$ is countable. We say that. a collection $\mathcal{B}$ of Borel subsets of $\mathbb{K}$ is a Borel subbase for $\mathbb{K}$ if and only if for every open $W \subseteq \mathbb{K}$ and every $\alpha \in W$, there are $B_{1}, \ldots, B_{m}$ in $\mathcal{B}$ with $\alpha \in \bigcap_{k=1}^{m} B_{k} \subseteq W$.

THEOREM 2. $f \in \mathcal{M}(\mu, X)$ if there is a $\mu$-covering set $\Sigma_{c}$ such that every $T \in$ $\Sigma_{c}$ admits pseudo-compact sets $P_{1}, P_{2}, \ldots$ in $X_{\sigma}, \mu$-null sets $N_{0}, N_{1}, \ldots$ with

$$
f\left(T \backslash N_{0}\right) \subseteq \overline{s p a n}\left(\bigcup_{i=1}^{\infty} f\left(T \backslash N_{i}\right) \cap P_{i}\right)=: Y,
$$

and a $U \subseteq X^{*}$ separating points of $Y$ with $U \circ f \subseteq \mathcal{M}(\mu)$ satisfying, for every $k \geqslant 1$, one of these conditions:

(1) There is a sequence $V_{k m}$ of pseudo-compact CCC-subspaces of $C\left(P_{k \sigma}\right)$ with $\left.U\right|_{P_{k}} \subseteq A l g\left(\bigcup_{m=1}^{\infty} V_{k m}\right)$. 
(2) There is a Borel subbase $\mathcal{B}_{k}$ for $\mathbb{K}$ so that every pairwise disjoint collection in

$\left\{T \cap \bigcap_{i=1}^{m} f^{-1}\left(P_{k} \cap u_{i}^{-1}\left(B_{i}\right)\right) \backslash N_{k} \mid m \in \mathbb{N}, u_{i} \in U, B_{i} \in \mathcal{B}_{k}\right\}$

is countable.

(3) $T \cap f^{-1}\left(P_{k}\right)$ is in $\widehat{\Sigma}$ and $\sigma$-finite; and there is a $\mu$-topology $\mathcal{T}_{k}$ on $T \cap$ $f^{-1}\left(P_{k}\right)$ such that $u \circ f$, restricted to $\left(T \cap f^{-1}\left(P_{k}\right)\right) \backslash N_{k}$, is $\mathcal{T}_{k}$-continuous for $u \in U$.

Conversely, these conditions are necessary for $f \in \mathcal{M}(\mu, X)$.

PROOF: Suppose that there exists a $\mu$-covering collection $\Sigma_{c}$ with the properties listed above. Fix $T \in \Sigma_{c}$, and then let the $P_{j}$ 's, the $N_{j}$ 's and $U$ be as guaranteed by the hypotheses. In view of Theorem 1 , it suffices to show that, for every fixed $k \geqslant 1$, the weak closure $C_{k}$ of $f\left(T \backslash N_{k}\right) \cap P_{k}$ is separable with respect to the weak topology. Note that $C_{k} \subseteq Y$, as $Y$ is closed in $X_{\sigma}$ by Mazur's Theorem [9, 17.1]. The class of Eberlein compacta (that is, those topological spaces that are homeomorphic with weakly compact subsets of Banach spaces) is well-known to be closed under the formation of countable products. And according to a result of Preiss and Simon [13], every weakly pseudo-compact subset of a Banach space is weakly compact. Thus, as $X$ admits a linear homeomorphic embedding into a product of countably many Banach spaces, $P_{k \sigma}$ is Eberlein compact.

First, suppose that (1) is satisfied. Fix $m$. Evaluation $g: V_{k m} \times P_{k \sigma} \rightarrow \mathbb{K}$ is separately continuous. Let $h \in C(\mathbb{K}, \mathbb{I})$. Then an argument given by Pták $[14, \mathrm{~F} .572]$ shows that

$$
\lim _{i \rightarrow \infty} \lim _{j \rightarrow \infty} h\left(v_{i}\left(p_{j}\right)\right)=\lim _{j \rightarrow \infty} \lim _{i \rightarrow \infty} h\left(v_{i}\left(p_{j}\right)\right) \text { in II }
$$

for every sequence $\left(v_{i}, p_{i}\right)$ in $V_{k m} \times P_{k}$ for which all limits involved exist. According to $\left[5,2.1\right.$.(2)], therefore, $h \circ g: V_{k m} \times P_{k \sigma} \rightarrow \mathbb{I}$ admits a separately continuous extension to $\beta V_{k m} \times P_{k \sigma}$, where $\beta$ denotes the Stone-Čech compactification operator. Furthermore, if $A, B$ are any two disjoint closed subsets of $\mathbb{K}$, then $h^{\prime}(A) \subseteq\{0\}$ and $h^{\prime}(B) \subseteq\{1\}$ for a suitable $h^{\prime} \in C(\mathbb{K}, \mathbb{I})$. Consequently, $g$ admits a separately continuous extension $\beta V_{k m} \times P_{k \sigma} \rightarrow \beta \mathbb{K}$ by [5, 2.1.(7)]. Moreover, all subspaces of $\mathbb{K}$ of the form $V_{k m}(p)$ with $p \in P_{k}$ and of the form $v\left(P_{k}\right)$ with $v \in V_{k m}$ are compact. It therefore follows from $[5,2.5 .(1), 2.8]$ that $V_{k m}$ has Eberlein compact closure $\bar{V}_{k m}$ in $C\left(P_{k \sigma}\right)$. On the other hand, $\bar{V}_{k m}$ satisfies CCC. Consequently, $\bar{V}_{k m}$ is separable. This follows from the Rosenthal Separability Theorem $[15,4.6]$ and is also a rather direct consequence of Namioka's continuity Theorem 4.2 in [11]. Thus, $A:=A \lg \left(\bigcup_{m=1}^{\infty} V_{k m}\right)$ is separable in 
$C\left(P_{k \sigma}\right)$, whence $\left.A\right|_{C_{k}}$ is separable in $C\left(C_{k \sigma}\right)$. But $\left.A\right|_{C_{k}}$ separates the points of $C_{k}$ since it contains $\left.U\right|_{C_{k}}$. Consequently, $C_{k \sigma}$ is separable, indeed.

In cases (2), (3), it suffices to show that $C_{k \sigma}$ satisfies CCC because it is Eberlein compact. So let $\left(Q_{\lambda}\right)_{\lambda \in \Lambda}$ be any family of non-empty, open, pairwise disjoint subsets of $C_{k \sigma}$. Fix $\lambda \in \Lambda$, and choose some $t_{\lambda} \in T \cap\left(f^{-1}\left(P_{k} \cap Q_{\lambda}\right) \backslash N_{k}\right)$. Since $\left.U\right|_{C_{k}}$ separates the points of $C_{k}$, it generates the topology of $C_{k \sigma}$. So there exist $u_{1}, \ldots, u_{m} \in U$ and open subsets $B_{1}, \ldots, B_{m}$ in $\mathbb{K}$ such that

$$
f\left(t_{\lambda}\right) \in C_{k} \cap \bigcap_{i=1}^{m} u_{i}^{-1}\left(B_{i}\right) \subseteq Q_{\lambda} .
$$

But then $f\left(D_{\lambda}\right) \subseteq Q_{\lambda}$ for

$$
D_{\lambda}:=T \cap f^{-1}\left(P_{k}\right) \cap \bigcap_{i=1}^{m}\left(u_{i} \circ f\right)^{-1}\left(B_{i}\right) \backslash N_{k} .
$$

And $D_{\lambda} \neq \emptyset$, as $t_{\lambda} \in D_{\lambda}$.

Suppose now that (3) holds. Then $D_{\lambda}$ is an open $\mathcal{T}_{k}$-neighbourhood of $t_{\lambda}$ in $\left(T \cap f^{-1}\left(P_{k}\right)\right) \backslash N_{k}$. Since $\mathcal{T}_{k}$ is a $\mu$-topology on $T \cap f^{-1}\left(P_{k}\right)$, it follows that $D_{\lambda} \notin \Sigma_{0}$, provided that $D_{\lambda} \neq\left(T \cap f^{-1}\left(P_{k}\right)\right) \backslash N_{k}$. Moreover, in view of the hypothesis that $U \circ f \subseteq \mathcal{M}(\mu)$ and that $T \cap f^{-1}\left(P_{k}\right)$ is $\sigma$-finite in $\widehat{\Sigma}$, we obtain that $D_{\lambda} \in \widehat{\Sigma}$ (compare $[2$, p.148]). Consequently, the usual summability argument shows that $\Lambda$ must be countable.

Finally, suppose that (2) is satisfied. Then we may assume that $B_{1}, \ldots, B_{m}$ are, actually, members of $\mathcal{B}_{k}$ (though no longer open, perhaps). Consequently, (2) guarantees countability of $\Lambda$ in this case as well.

Conversely, as for the necessity of the conditions, suppose that $f$ is $\mu$-measurable. Take $\Sigma_{c}:=\Sigma_{b}$ and $U:=X^{*}$. Fix $T \in \Sigma_{c}$ and, thereafter, $N_{0} \in \Sigma_{0}$ such that $\bigcup_{i=1}^{\infty} P_{i}$ is dense in $f\left(T \backslash N_{0}\right)$ for some sequence $P_{k}$ of singletons. Let $N_{k}:=\emptyset$ for $k \geqslant 1$. Then (1) and (2) are, trivially, satisfied for every $k$. As is (3) since $T \cap f^{-1}\left(P_{k}\right) \in \widehat{\Sigma}$ (compare [2, p.148]).

Remarks. 1. The proof of Theorem 1 essentially consisted in showing that, if $U \circ f \subseteq$ $\mathcal{M}(\mu)$ for some $U \subseteq X^{*}$ separating the points of $X$, then $f$ is scalarly $\mu$-measurable, provided that $X$ is separable. Scalar measurability has received considerable attention, in particular in the context of the Pettis integral (compare [18] and references there). The Krein-Šmulian Theorem can be applied to the scalar measurability problem as well. Even if $X$ is non-separable, the argument for scalar $\mu$-measurability of $f$ in the proof of Theorem 1 goes through, provided that $\left(B^{\circ}\right)_{\sigma}$ is sequential for every 0 neighbourhood $B$ in $X$. The latter condition is satisfied, for instance, if $X$ is a closed 
linear subspace of a Fréchet space $Z$ such that $\bigcup_{n=1}^{\infty} P_{n}$ is total in $Z$ for some sequence $P_{n}$ of pseudo-compact subsets of $Z_{\sigma}$. But some additional assumption on $X$ is, generally, indispensable to arrive at the conclusion. Consider, for instance, the map $g: \mathbb{I} \rightarrow \ell^{\infty}$, where $g(s)$ is the sequence making up the dyadic expansion of $s \in \mathbb{I}$ such that $g(s)$ is not 1 eventually. Let $U \subseteq\left(\ell^{\infty}\right)^{*}$ consist of the evaluation functionals at points of $\mathbb{N}$. For every $u \in U$, then $u \circ g$ is measurable with respect to Lebesgue measure $\lambda$ (in fact, $u \circ g$ is of Baire class 1 ). It can be shown, however, that $g$ is not scalarly $\lambda$-measurable. 2. If $S$ is a Souslin space and $\mu$ a Borel measure, then the set $\mathcal{K}(S)$ of all compact subsets of $S$ is a $\mu$-regularity set (compare [16, p.122]).

3. If $S$ is a completely regular space and $\mu$ a Baire measure, then it can be shown that the collection $\mathcal{Z}(S)$ of zero-sets of $S$ (that is, sets $g^{-1}(0)$ with $g \in C(S, \mathbb{I})$ ) is a $\mu$-regularity set. This, in turn, can be used to show that, if $S$ is a locally compact group and $\mu$ a Haar measure, then $\mathcal{Z}(S) \cap \mathcal{K}(S)$ is a $\mu$-regularity set; if, moreover, the connected components of $S$ are metrisable, then those members of $\mathcal{Z}(S)$ that are homeomorphic with $\{0,1\}^{w}$ form a $\mu$-regularity set, where $w$ is the local weight of $S$ [6, p.337].

4. In Theorems $1,2, \quad U \circ f \subseteq \mathcal{M}(\mu)$ can be replaced by $\left.U \circ f\right|_{T} \subseteq \mathcal{M}\left(\mu_{T}\right)$, where $\mu_{T}$ is the restriction of $\mu$ to $T$.

5. For certain measures $\mu$, a much stronger version of the $\sigma$-compactness condition for $f\left(T \backslash N_{0}\right)$ in Theorem 2 is a necessary by-product of $\mu$-measurability. Suppose $S$ is also a topological space and has a $\mu$-regularity set $\Gamma$ consisting of closed pseudo-compact sets of $S$. (For example, $\mu$ a Radon measure.) If $f \in \mathcal{M}(\mu, X)$, then every $T \in \Sigma_{b}$ admits some $N_{0} \in \Sigma_{0}$ such that $f\left(T \backslash N_{0}\right)$ is $\sigma$-compact, even in $X$. This follows from the fact (compare the Example) that $T$ contains, for every $\varepsilon>0$, some $G \in \Gamma$ with $\mu(T \backslash G)<\varepsilon$ such that $\left.f\right|_{G}: G \rightarrow X$ is continuous.

6. Given the $P_{k}$ 's in Theorem 2, condition (1) is satisfied if $U$ is pointwise bounded on $X$ and $U_{\sigma}$ satisfies CCC. For instance, if $Z$ is a Banach space for which $B:=\{z \in Z \mid$ $\|z\| \leq 1\}_{\sigma}$ satisfies CCC - such spaces have been studied and examples exhibited by Wheeler in [20] - and if $X$ is the strong dual of $Z$, then one may take the set $U \subseteq X^{*}$ corresponding to $B$ or any sufficiently large subset thereof.

7. Concerning the countable chain condition in (1), it would not be good enough to suppose that $\bigcup_{m=1}^{\infty} V_{k m}$ be a CCC-space. Consider $X:=\ell^{2}(\mathbb{I}), U:=X^{*}, P_{k}:=$ $\{x \mid\|x\| \leqslant 1\}$, and $V_{k m}:=\left\{v\left|P_{k}\right| v \in X^{*},\|v\| \leqslant m\right\}$. Since $X_{\sigma}^{*}$ is homeomorphic with a dense subset of some product $\mathbb{K}^{\Lambda}$, it satisfies CCC. But the scalarly Lebesgue measurable function $f: s \mapsto \chi_{\{a\}}: \mathbb{I} \rightarrow X$ is not measurable (as Birkhoff observed long ago).

8. In Theorem 2: if $T \cap f^{-1}\left(P_{k}\right)$ is in $\widehat{\Sigma}$ and $\sigma$-finite and the set system in (2) has 
only $\emptyset$ in common with $\Sigma_{0}$ for some $\mathcal{B}_{k}$, then (2) is satisfied.

9. If (3) is satisfied for all $k$, if $T \backslash \bigcup_{k=1}^{\infty} f^{-1}\left(P_{k}\right) \in \Sigma_{0}$, and $\bigcup_{k=1}^{\infty} \mathcal{T}_{k} \subseteq \widehat{\Sigma}$, then the hypothesis $U \circ f \subseteq \mathcal{M}(\mu)$ is redundant since $\left.U \circ f\right|_{T} \subseteq \mathcal{M}\left(\mu_{T}\right)$ in this situation.

10. Let $\mathcal{F}$ be the class of those Fréchet spaces in which all weakly compact, hence all weakly pseudo-compact, subsets are separable. (1), (2), (3) in Theorem 2 are redindant if $X \in \mathcal{F}$. - Now let $X=L^{\infty}(\nu, Y)$, where $\nu$ is a $\sigma$-finite measure and $Y$ a Banach space. Clearly, $X \in \mathcal{F}$, if $Y \in \mathcal{F}$ and $L^{1}(\nu)$ is separable. It is shown in [7] that large subspaces of $X$ are in $\mathcal{F}$ if $Y$ admits a continuous linear injection into some Banach space $Z$ such that $W_{\sigma}$ satisfies CCC for some bounded $W \subseteq Z^{*}$ separating the points of $Z$. More elementary is the fact that $X \in \mathcal{F}$ if $Y_{\sigma}^{*}$ is separable. For a compact space $L$, the Banach space $C(L)$ may have a weak*-separable dual, even when $L$ itself is not separable; compare [10] and [17]. In [3, 5.6], an example is given of a Banach space $X$ with $X_{\sigma}^{*}$ separable (equivalently: with a countable subset of $X^{*}$ separating the points of $X$ ) such that no countable subset of $X^{*}$ is norming.

Corollary. Let $\mu$ be $\sigma$-finite. If $f \in \mathcal{M}(\mu, X)$, then there are $N \in \Sigma_{0}$, some $U \subseteq X^{*}$ separating points of $\overline{\operatorname{span}} f(S \backslash N)$, and a topology $\mathcal{T}$ on $S$ with $\mathcal{T} \backslash\{\emptyset\} \subseteq \widehat{\Sigma} \backslash \Sigma_{0}$ rendering $\left.u \circ f\right|_{S \backslash N} \mathcal{T}$-continuous for all $u \in U$. The converse holds if $f(S \backslash M)$ is relatively compact in $X_{\sigma}$ for some $M \in \Sigma_{0}$.

Proof: Let $f \in \mathcal{M}(\mu, X)$ and then $Z$ a separable closed linear subspace of $X$ such that $N_{1}:=f^{-1}(X \backslash Z) \in \Sigma_{0}$. Choose a countable subset $U$ of $X^{*}$ that separates the points of $Z$. Moreover, let $\theta$ be a lifting of $\widehat{\Sigma}$. (Such a lifting exists: if $(S, \Sigma, \mu)$ is not finite and complete, instead we may consider any measure $\nu: \widehat{\Sigma} \rightarrow \mathbb{I}$ producing the same null sets as $\mu$. Compare $[8$, p.46].) But then every $u \in U$ admits some $N_{u} \in \Sigma_{0}$ for which $\left.u \circ f\right|_{S \backslash N_{u}}$ is continuous with respect to the density topology $T_{\theta}$ on $S\left[8\right.$, p.59]. Consequently, $N:=N_{1} \cup \bigcup_{u \in U} N_{u}$ does the job. Conversely, suppose that, in addition to the conditions listed, there exist a weakly compact subset $P$ of $X$ and some $M \in \Sigma_{0}$ such that $f(S \backslash M) \subseteq P$. Then the $\mu$-measurability of $f$ follows by means of Theorem 2.(3) with $N_{0}:=N \cup M=: N_{k}, \Sigma_{c}:=\left\{S \backslash N_{0}\right\}, P_{k}:=P$, and $\mathcal{T}_{k}:=\left\{Q \backslash N_{0} \mid Q \in \mathcal{T}\right\}$; compare Remark 9 .

\section{REFERENCES}

[1] J. Diestel and J.J. Uhl, jr, Vector measures, Mathematical Surveys 15 (American Mathematical Society, Providence, R.I. 1977).

[2] N. Dunford and J.T. Schwartz, Linear operators I (Interscience Publ., New York, 1958).

[3] G.A. Edgar, 'Measurability in a Banach space II', Indiana Univ. Math. J. 28 (1979), 559-579. 
[4] D. Helmer, 'Continuity of locally compact group actions with measurable orbit maps', Math. Z. 172 (1980), 51-53.

[5] D. Helmer, 'Criteria for Eberlein compactness in spaces of continuous functions', Manuscripta Math. 35 (1981), 27-51.

[6] D. Helmer, 'Structure of locally compact groups with metrizable connected components up to negligible subsets', Arch. Math. 51 (1988), 332-342.

[7] D. Helmer, Weakly compact and separable subsets of $L^{\infty}(\mu, X)$, (in preparation).

[8] A. Ionescu Tulcea and C. Ionescu Tulcea, Topics in the theory of lifting (Springer-Verlag, Berlin, Heidelberg, New York, 1969).

[9] J.L. Kelley, I. Namioka, Linear topological spaces (D. Van Nostrand, Princeton, N.J., 1963).

[10] G. Mägerl and I. Namioka, 'Intersection numbers and weak" separability of spaces of measures', Math. Ann. 249 (1980), 273-279.

[11] I. Namioka, 'Separate continuity and joint continuity', Pacific J. Math. 51 (1974), 515-531.

[12] B.J. Pettis, 'On integration in vector spaces', Trans. Amer. Math. Soc. 44 (1938), 277-304.

[13] D. Preiss and P. Simon, 'A weakly pseudocompact subspace of Banach space is weakly compact', Comment. Math. Univ. Carolinae 15 (1974), 603-609.

[14] V. Pták, 'An extension theorem for separately continuous functions and its application to Functional Analysis', Czechoslovak Math. J. 14 (1964), 562-571.

[15] H.P. Rosenthal, 'On injective Banach spaces and the spaces $L^{\infty}(\mu)$ for finite measures н', Acta Math. 124 (1970), 205-248.

[16] L. Schwartz, Radon measures on arbitrary topological spaces and cylindrical measures (Oxford University Press, Bombay, 1973).

[17] M. Talagrand, 'Separabilite vague dans l'espace des measures sur un compact', Israel J. Math. 37 (1980), 171-180.

[18] M. Talagrand, Pettis integral and measure theory, Memoirs 307 (American Mathematical Society, Providence, R.I., 1984).

[19] J.J. Uhl, jr., 'Pettis's measurability theorem', Contemp. Math. 2 (1980), 135-144.

[20] R.F. Wheeler, 'The retraction property, CCC property, and Dunford-Pettis-Phillips property for Banach spaces', in Proc. Measure Theory Conf., Oberwolfach, Lecture Notes in Math. 945 (Springer-Verlag, Berlin, Heidelberg, New York, 1981), pp. 252-262.

\footnotetext{
Department of Mathematics

University of Bahrain

PO Box 32038

Isa Town

Bahrain
} 